Canadian Journal of Applied Linguistics

Revue canadienne de linguistique appliquée

\title{
Niveaux de compétence linguistique canadiens et les outils connexes : une proposition pédagogique raisonnée en FLS pour immigrants adultes en contexte francophone minoritaire
}

\author{
Morgan Le Thiec, Monika Jezak and Élissa Beaulieu
}

Volume 21, Number 1, 2018

URI: https://id.erudit.org/iderudit/1057921ar

DOI: https://doi.org/10.7202/1057921ar

See table of contents

\section{Publisher(s)}

University of New Brunswick

ISSN

1920-1818 (digital)

Explore this journal

Cite this article

Le Thiec, M., Jezak, M. \& Beaulieu, É. (2018). Niveaux de compétence linguistique canadiens et les outils connexes : une proposition pédagogique raisonnée en FLS pour immigrants adultes en contexte francophone minoritaire. Canadian Journal of Applied Linguistics / Revue canadienne de linguistique appliquée, 21(1), 123-140. https://doi.org/10.7202/1057921ar

\section{Article abstract}

The purpose of this article is to present the pedagogical proposal that underlies the development of tools for the language training of adult immigrants in Francophone minority contexts. These tools complement the Niveaux de compétence linguistique canadiens (NCLC) 2012 framework (Citizenship and Immigration Canada and Centre for Canadian Language Benchmarks, 2012). In our article, we first sketch the problem of teaching French as a second language to adult immigrants in a minority setting. Second, we explain the research design that led to the development of the NCLC 2012, considering language learning and the contextual constraints in which the framework and its associated tools are used. Third, we give some examples of tools adapted to the teaching of French as a second language in minority Francophone communities that follow the pedagogical approach proposed by the NCLC. To conclude, we take stock of many challenges that remain in the development of tools for our target clientele.
Copyright (c) Morgan Le Thiec, Monika Jezak, Élissa Beaulieu, 2018

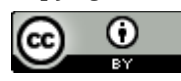

This document is protected by copyright law. Use of the services of Érudit (including reproduction) is subject to its terms and conditions, which can be viewed online.

https://apropos.erudit.org/en/users/policy-on-use/ 


\title{
Niveaux de compétence linguistique canadiens et les outils connexes : une proposition pédagogique raisonnée en FLS pour immigrants adultes en contexte francophone minoritaire
}

\author{
Morgan Le Thiec \\ Université du Québec à Montréal \\ Monika Jezak \\ Institut des langues officielles et du bilinguisme, Université d'Ottawa \\ Élissa Beaulieu \\ Centre for Canadian Language Benchmarks
}

\begin{abstract}
Résumé
L'objectif de cet article est de présenter la proposition pédagogique qui sous-tend depuis quelques années le développement des outils destinés à la formation langagière des immigrants adultes en contexte francophone minoritaire. Ces outils complémentent le référentiel Niveaux de compétence linguistique canadiens (NCLC) 2012 (Citoyenneté et Immigration Canada et Centre des niveaux de compétence linguistique canadiens, 2012). Dans notre article, dans un premier temps, nous esquissons la problématique de l'enseignement du français langue seconde aux immigrants adultes en milieu minoritaire. Dans un deuxième temps, nous expliquons le plan de recherche qui a permis l'élaboration des NCLC 2012 en tenant compte à la fois des acquis en didactique des langues et des contraintes du contexte dans lequel le référentiel et ses outils connexes sont utilisés. Dans un troisième temps, nous donnons quelques exemples d'outils adaptés à l'enseignement du français langue seconde en milieu francophone minoritaire qui suivent l'approche pédagogique proposée par les NCLC. En conclusion de l'article, nous faisons le point sur de nombreux défis qu'il reste à relever dans le cadre du développement des outils destinés à notre clientèle cible.
\end{abstract}

\begin{abstract}
The purpose of this article is to present the pedagogical proposal that underlies the development of tools for the language training of adult immigrants in Francophone minority contexts. These tools complement the Niveaux de compétence linguistique canadiens (NCLC) 2012 framework (Citizenship and Immigration Canada and Centre for Canadian Language Benchmarks, 2012). In our article, we first sketch the problem of teaching French as a second language to adult immigrants in a minority setting. Second, we explain the research design that led to the development of the NCLC 2012, considering language learning and the contextual constraints in which the framework and its associated tools are used. Third, we give some examples of tools adapted to the teaching of French as a second language in minority Francophone communities that follow the pedagogical approach proposed by the NCLC. To conclude, we take stock of many challenges that remain in the development of tools for our target clientele.
\end{abstract}


Niveaux de compétence linguistique canadiens et les outils connexes : une proposition pédagogique raisonnée en FLS pour immigrants adultes en contexte francophone minoritaire

\section{Introduction}

En 2002, le Centre des niveaux de compétence linguistique canadiens (CNCLC) produisait la première version française des Canadian Language Benchmarks (CLB): English as a Second Language for Adults (working document, 1996) [Citoyenneté et Immigration Canada, 1996]. Ce référentiel, intitulé Standards linguistiques canadiens 2002 : français langue seconde pour adultes (Citoyenneté et Immigration Canada et Centre des niveaux de compétence linguistique canadiens, 2002) fut révisé en 2006 et renommé Niveaux de compétence linguistique canadiens (NCLC) 2006 : français langue seconde pour adultes (Citoyenneté et Immigration Canada et Centre des niveaux de compétence linguistique canadiens, 2006).

C'est la dernière version des NCLC (Citoyenneté et Immigration Canada et Centre des niveaux de compétence linguistique canadiens, 2012) qui sert aujourd'hui de référence lors de la création d'outils, de ressources et de formations en français langue seconde (FLS) pour adultes en milieu francophone minoritaire.

L'objectif de cet article est donc un examen de la proposition pédagogique qui découle des NCLC. Dans un premier temps, nous décrivons brièvement la problématique qui est sous-jacente au développement des outils pédagogiques relatifs aux NCLC : celle des défis et des enjeux de l'enseignement du FLS aux immigrants adultes en milieu francophone minoritaire. Dans un deuxième temps, nous présentons le plan de recherche qui a abouti à la version 2012 des NCLC et qui sous-tend le développement des outils pédagogiques. Nous présentons ensuite certains projets récemment complétés et ceux en cours qui illustrent la démarche pédagogique proposée. À la suite de ce tour d'horizon rapide, nous faisons le point sur de nombreux défis qu'il reste à relever dans le cadre du développement des outils destinés à la formation langagière des immigrants adultes en contexte francophone minoritaire.

\section{Éléments de la problématique : enjeux et défis de la formation langagière pour les immigrants adultes en milieu francophone minoritaire}

\section{Immigration au sein des communautés francophones minoritaires}

L'immigration ${ }^{1}$ constitue, de nos jours, le principal moteur de croissance démographique et économique du Canada (Martel, 2015). Cependant, presque tous les nouveaux arrivants hors Québec s'installent chaque année au sein des communautés majoritaires anglophones, menaçant ainsi la vitalité des communautés francophones minoritaires (Chong, 2015 ; Corbeil, Grenier et Lafrenière, 2006 ; Houle, Pereira et Corbeil, 2015). Comme l'ont constaté Fraser et Boileau (2014) :

Les communautés francophones non seulement connaissent des baisses marquées de natalité et des taux accrus de vieillissement, mais encore sont touchées par des questions de transferts linguistiques et de transmission intergénérationnelle de la langue vers l'anglais, ce qui avec le temps influe sur leur vitalité sur le plan 
démographique. L’immigration représente donc une condition essentielle à leur essor. Or, dans l'ensemble, les communautés francophones ... ont peu bénéficié de l'apport démographique de l'immigration internationale en raison de la forte propension de ces immigrants à s'intégrer aux communautés majoritaires d'expression anglaise. (p. 8)

Face à cette réalité, depuis une dizaine d'années, le gouvernement canadien produit des législations qui lui permettent d'allouer des fonds substantiels destinés à encourager les immigrants récents à s'installer au sein des communautés francophones en situation minoritaire. Parmi ces législations, on peut nommer le Cadre stratégique pour favoriser l'immigration au sein des communautés francophones en situation minoritaire (Gouvernement du Canada, 2003) ; le Plan stratégique pour favoriser l'immigration au sein des communautés francophones en situation minoritaire (Gouvernement du Canada, 2006) ; la Feuille de route pour la dualité linguistique 2008-2013 (Gouvernement du Canada, 2008) ; le Plan d'action 2009-2013 : mise en ouvre de l'article 41 de la Loi sur les langues officielles (Gouvernement du Canada, 2009); et la Feuille de route pour les langues officielles du Canada 2013-2018 : éducation, immigration, communautés (Gouvernement du Canada, 2013).

Les immigrants de langue maternelle française sont visés prioritairement par les politiques ci-dessus énumérées, mais le gouvernement encourage également les immigrants d'expression française qui pourraient choisir le français comme première langue officielle ${ }^{2}$ de par leur éducation antérieure en français, de par un séjour prolongé dans un pays où la langue officielle était le français, ou simplement, de par leurs affinités culturelles déclarées avec la langue et les cultures francophones. Pour ces immigrants-là, l'amélioration des compétences en français est une condition préalable à leur insertion au sein des communautés francophones (Jezak et Carrasco, 2017).

Depuis les années 2008-2009, les besoins langagiers des nouveaux arrivants francophones ou francophiles sont explicitement reconnus dans les énoncés de politiques d'immigration. Autant le ministère fédéral de l'Immigration, Réfugiés et Citoyenneté (Plan d'action 2009-2013) que le ministère de Patrimoine canadien (Feuille de route pour la dualité linguistique 2008-2013) ont été dotés de nouvelles mesures législatives ainsi que de fonds destinés expressément aux immigrants désirant s'installer au sein des communautés francophones en situation minoritaire. Plus récemment, la Feuille de route pour les langues officielles du Canada 2013-2018 : éducation, immigration, communautés (Gouvernement du Canada, 2013), élaborée à la suite d'une consultation pancanadienne parmi les plus importantes jamais organisées sur les langues officielles, a affirmé que l'immigration continuait à être un des secteurs prioritaires des politiques linguistiques en milieu minoritaire. Tel que l'ont constaté Cardinal, Gaspard et Léger (2015), le point focal de la Feuille de route 2013-2018 était la valeur unificatrice des deux langues officielles et la valeur économique de l'immigration («avantage concurrentiel du Canada tant à l'échelle nationale qu'internationale », Gouvernement du Canada, 2013, p. 1). Concrètement, cette nouvelle politique s'est traduite par un investissement de 149,5 millions de dollars dans les programmes de langues officielles à l'intention des immigrants. 


\section{Formation en FLS pour immigrants adultes en contexte minoritaire}

Toutefois, la reconnaissance officielle de la langue française, celle des droits des francophones minoritaires et de l'importance de l'immigration d'expression française, ainsi que l'attribution de fonds pour les cours de FLS en milieu minoritaire ne règlent pas tout. Dans les faits, le français hors Québec reste une langue largement minoritaire (environ 1 million de locuteurs selon Corbeil, 2014). De plus, alors que l'accessibilité de facto à des services offerts en français dépend de la concentration de francophones sur un territoire donné, les minorités francophones sont réparties à travers tout le Canada et regroupées en communautés dont le nombre d'individus varie beaucoup d'une région à l'autre et dont la population est parfois très dispersée, créant des situations de fragilité démo-linguistique (Houle et coll., 2015 ; Quell, 2008).

Les NCLC et les services linguistiques associés, créés dans ce contexte particulier, doivent donc répondre à un double objectif : (a) permettre aux immigrants adultes qui le souhaitent de vivre et de travailler en français, mais aussi (b) renforcer les communautés francophones en facilitant l'intégration des immigrants.

Afin de répondre à ce double objectif, lors de la création de programmes et d'outils de formation langagière pour immigrants adultes, il est essentiel de prendre en compte les besoins et les intérêts particuliers (et très variés) de différents acteurs : les communautés d'accueil, les immigrants adultes désireux de s'y intégrer et les intervenants en formation. En conséquence, les formations en FLS en milieu minoritaire sont, par principe, bâties au cas par cas.

Dans la suite de notre article, nous traitons des besoins et des attentes des parties qui définissent directement les choix pédagogiques : les immigrants-apprenants et leurs formateurs.

Immigrants-apprenants. Les immigrants adultes qui souhaitent entreprendre une formation langagière financée par le gouvernement fédéral peuvent choisir entre l'anglais et le français (www.cic.gc.ca). Plus précisément, ils ne peuvent pas suivre concurremment les cours dans les deux langues comme en témoignent les critères d'éligibilité présentés sur le site Internet settlement.org associé à la province de l'Ontario (settlement.org).

Logiquement, cette réalité peut avoir un impact sur les inscriptions du côté français hors Québec, puisque l'immigrant adulte s'implique dans des projets qui lui permettront de s'intégrer le plus vite possible dans la société canadienne. C'est le cas, par exemple, des immigrants qui déclarent avoir comme première langue officielle parlée le français et l'anglais et qui privilégient l'anglais dans leur vie de tous les jours (Houle et coll., 2015). Les raisons qui sont à la base du choix d'apprendre le français doivent donc être d'ordre différent. Nous les présentons à titre d'hypothèses issues d'échanges avec les formateurs et les administrateurs lors de rencontres en présentiel ou en ligne. Les analyses des besoins réalisées auprès des apprenants au début des formations ont révélé qu'il pouvait s'agir, par exemple, de l'attrait de la vie communautaire en milieu francophone. Certains immigrants voulaient inscrire leurs enfants dans des écoles francophones, suivre leur scolarité en français et profiter des services francophones disponibles. Pour les immigrants qui maîtrisaient suffisamment bien l'anglais, il pouvait s'agir également de projets professionnels reliés aux services fédéraux ou autres qui favorisaient le bilinguisme. Une autre source de motivation pouvait être une bonne compréhension du contexte linguistique 
du Canada : Contrairement au français, l'anglais peut s'apprendre en dehors d'une classe puisqu'il s'agit de la langue dominante dans la vie de tous les jours.

Formateurs. Pour leur part, les postures des formateurs ont été examinées dans le cadre du projet Perceptions des intervenants en français langue seconde au sujet de l'utilisation des NCLC version 2006 (Dancose et Ricard, 2007). Par ce projet, les chercheurs ont voulu vérifier dans quelle mesure les NCLC et leurs outils connexes répondaient aux deux grandes fonctions des standards de formation conceptualisées par Klieme, Avenarius, Blum, Döbrich et Vollmer (2004). D'une part, les standards devaient fournir des repères précis aux intervenants responsables des formations (gestionnaires, formateurs) pour orienter leurs décisions et pratiques à partir d'un langage commun et d'une compréhension partagée de la progression des apprentissages. De l'autre part, leur seconde fonction était de permettre l'évaluation des résultats obtenus par les apprenants et d'offrir les conditions nécessaires à une reddition de compte constructive autant pour les responsables des formations que pour les publics.

Les groupes de discussion organisés dans le cadre de cette consultation pancanadienne avaient pour tâche d'échanger sur les sujets suivants : les programmes et pratiques d'intervention, les besoins des apprenants et intervenants, le niveau d'intérêt à adopter les NCLC, les étapes d'implantation des NCLC.

Parmi les préoccupations concrètes des participants à ces groupes de discussion, on mentionnait des difficultés de transfert d'apprenants d'un établissement à l'autre, les programmes n'étant pas standardisés. L'absence d'outils d'évaluation fiables ne permettait pas non plus de mesurer efficacement les progrès des apprenants, de les orienter vers des formations appropriées ou encore d'évaluer leur compétence en français du point de vue de leur intégration au marché du travail. Enfin, les intervenants interrogés se préoccupaient du modèle de formation linguistique le plus pertinent dans un contexte où le français était une langue minoritaire.

Sur le plan d'applications possibles des NCLC, les participants aux groupes de discussions se sont entendus sur plusieurs points :

- Utiliser les NCLC comme base reconnue au niveau national : de cette façon, un niveau de compétence serait le même d'une province à l'autre. Un apprenant formé au Manitoba et présentant un NCLC 5 serait capable de réaliser les mêmes tâches langagières qu'un apprenant formé au Nouveau-Brunswick et présentant lui aussi un niveau NCLC 5. Ainsi, un apprenant en formation linguistique pourrait migrer d'une province à l'autre et faire reconnaître son niveau NCLC au moment d'intégrer une nouvelle formation linguistique.

- Uniformiser les programmes de formation linguistique : cette uniformisation aurait l'avantage à la fois de faciliter la migration d'un public souvent mobile dans les premières années de séjour au Canada et de favoriser l'évaluation des programmes et les échanges entre intervenants.

- Répondre aux besoins des apprenants adultes : les descriptions présentées dans les NCLC permettraient aux apprenants de se situer sur un continuum de progression à partir de tâches langagières et de situations de communication clairement identifiées et pertinentes dans la vie de tous les jours. 
- Renforcer le lien entre la formation linguistique offerte avec les NCLC et l'emploi : ce renforcement permettrait d'aider les apprenants à prendre conscience de leurs besoins langagiers par rapport aux exigences de l'emploi ou de la profession qui les intéresse.

En réponse aux besoins du milieu de la formation exprimés dans le document Perceptions des intervenants en français langue seconde au sujet de l'utilisation des NCLC version 2006 (Dancose et Ricard, 2007), les NCLC ont été révisés de façon substantielle et plusieurs projets pédagogiques ont vu le jour. À l'heure actuelle, certains de ces projets sont déjà complétés et d'autres sont en cours de réalisation. De façon générale, ils ont pour vocation de soutenir différents acteurs du FLS (apprenants, formateurs, gestionnaires de programmes, agents d'insertion, etc.) en favorisant l'harmonisation des contenus et des pratiques. Comme recommandé à la suite des projets dont nous venons de rendre compte, ils proposent une démarche pédagogique unique. Plus globalement, ils s'inscrivent dans les efforts entrepris par Immigration, Réfugiés et Citoyenneté Canada afin de répondre aux exigences d'assurance-qualité dans le domaine de l'éducation (Harvey, 2008 ; Organisation de Coopération et de Développement Économiques, 2015).

Avant de faire un bref survol de ces projets, dans la section suivante de l'article nous présentons le plan de recherche qui a permis de bâtir le référentiel Niveaux de compétence linguistique canadiens 2012 et d'élaborer une proposition pédagogique pour l'enseignement du FLS en milieu minoritaire en tenant compte à la fois des acquis théoriques en didactique des langues et du contexte particulier de l'utilisation de ces outils.

\section{Méthodologie : le plan de recherche pour le développement des NCLC 2012 et de la proposition pédagogique connexe}

La méthodologie de recherche qui sous-tend le développement des NCLC et de la proposition pédagogique connexe s'inscrit dans le paradigme de recherche développement en éducation (Harvey et Loiselle, 2009 ; Legendre, 2005 ; Loiselle et Harvey, 2007 ; Van der Maren, 1995). Plus particulièrement, il s'agit du plan de type recherche design. ${ }^{3}$

Dans le domaine de l'éducation, les premiers projets de ce type datent du début des années 1990 (Brown, 1992 ; Collins, 1992) et ont été élaborés en réponse aux critiques des milieux éducatifs adressées à l'endroit de la recherche expérimentale classique qui «ne tient pas compte de la multiplicité des variables en cause dans des contextes réels d'apprentissage et d'enseignement, ni de la nature complexe et 'désordonnée' de ces contextes, et, de ce fait, ne conduit pas à des résultats qui permettent d'expliquer et de prédire l'apprentissage et de guider de manière utile les pratiques éducatives » (Basque, 2015, p. 1). En effet, selon la définition de Wang et Hannafin (2005), l'approche de recherche design fournit :

A systematic but flexible methodology aimed to improve educational practices through iterative analysis, design, development, and implementation, based on collaboration among researchers and practitioners in real-world settings, and leading to contextually-sensitive design principles and theories. (p. 6) 
Selon plusieurs auteurs (Cobb, Confrey, diSessa, Lehrer et Schauble, 2003 ; Dede, 2004 ; Design-Based Research Collective, 2003 ; Kelly, 2003 ; Reeves, Herrington et Oliver, 2005), la recherche design possède les cinq caractéristiques suivantes :

1. elle vise tout d'abord le développement d'une intervention pédagogique en réponse à un besoin identifié par des acteurs du milieu d'éducation ;

2. elle s'appuie sur une démarche itérative qui incorpore, en spirale, le design, l'évaluation et la révision du produit ;

3. elle est orientée vers une amélioration progressive de l'intervention à la suite de consultations avec les futurs usagers ;

4. sa valeur repose principalement sur la fonctionnalité du produit qui en résulte et

5. l'intervention proposée s'appuie sur une proposition théorique et, en retour, la validation du produit contribue au développement de la théorie. Aux cinq points cidessus énumérés s'ajoute le principe de faire appel, de façon systématique, à une variété d'approches et d'outils de recherche.

Bournot-Trites (2017) a décrit en détail l'application du paradigme de recherche design à l'élaboration du cadre théorique (Bournot-Trites, Barbour, Jezak, Stewart et Blouin Carbonneau, 2015) et des échelles des NCLC 2012 (Citoyenneté et Immigration Canada et Centre des niveaux de compétence linguistique canadiens, 2012), ainsi que les avantages d'utiliser cette approche pour le développement des interventions dans la cadre de la formation langagière pour adultes en milieu minoritaire (Bournot-Trites et Barbour, 2012 ; Elson, 2012). Afin d'illustrer brièvement de quelle façon les cinq principales caractéristiques de la recherche design s'appliquaient à la recherche sur les NCLC, considérons les points suivants.

En premier lieu, le développement de ce référentiel et des outils connexes découlait de recommandations faites à la suite de larges consultations menées auprès des enseignants de FLS aux adultes immigrants en milieu minoritaire et visait à répondre tout d'abord aux besoins identifiés par ces enseignants.

Dans un deuxième temps, la démarche itérative s'est traduite par de nombreux vaet-vient entre l'équipe de chercheurs, les différents experts de contenu et de politiques et les acteurs du milieu. Ces échanges ont eu lieu grâce aux groupes de réflexion, aux questionnaires et aux diverses consultations qui ont fait partie du plan de recherche, autant à la phase de développement qu'à la phase de validation. La fonctionnalité du produit (les échelles NCLC) a été la principale motivation pour le projet, mais le fait d'adopter la démarche itérative a eu également pour résultat le développement d'une communauté de pratique malgré des distances géographiques qui séparaient les différents lieux d'enseignement ainsi qu'une meilleure adaptation des outils, des ressources et des approches à une grande variété de scénarios pédagogiques. Nous reviendrons à ce point dans la conclusion de cet article.

Finalement, tel que le stipulent les principes de la recherche design, l'équipe de chercheurs chargée du développement des NCLC a trouvé indispensable d'assoir les échelles sur des bases théoriques solides. Un cadre théorique, inspiré des travaux de Bachman (Bachman, 1990 ; Bachman et Palmer, 1996 ; Bachman et Palmer, 2010) a été conçu et validé en amont de la conceptualisation des échelles.

L'application du paradigme de recherche design, tel que décrit dans Bournot-Tites (2017) et esquissé dans les paragraphes précédents, a conduit à l'instauration d'une 
proposition pédagogique propre aux NCLC qui reposait sur quatre principes complémentaires (Citoyenneté et Immigration Canada et Centre des niveaux de compétence linguistique canadiens, 2012, p. 2-3). Cette démarche était :

1. centrée sur les apprenants : les apprentissages sont tout d'abord pertinents pour l'apprenant et répondent à ses intérêts. Ils sont également adaptés à ses capacités langagières et à son style d'apprentissage ;

2. centrée sur les compétences : l'accent est mis sur l'application concrète des connaissances et non pas sur les connaissances linguistiques elles-mêmes ;

3. centrée sur les tâches : la notion de tâche langagière est centrale à l'enseignement dans la mesure où celui-ci implique l'utilisation de la langue dans un cadre de communication authentique, pour atteindre un objectif précis dans un contexte particulier ;

4. centrée sur des contextes de la collectivité, des études et du travail : on privilégie les tâches qui s'inscrivent dans des contextes de vie des apprenants où la communication nécessite des renseignements contextuels précis et des connaissances préalables.

Dans la suite de l'article, nous présentons quelques exemples de projets récents et ceux encore en cours qui illustrent la mise en pratique de ces principes. Nous avons regroupé ces projets en deux volets : les outils et ressources pour l'apprenant et les outils et ressources pour le formateur.

\section{De la recherche à la pratique : quelques outils et ressources pour la formation en FLS en milieu minoritaire}

\section{Outils pour les immigrants-apprenants}

Selon les informations échangées au sein des communautés de pratique et les analyses des besoins réalisées auprès des apprenants au début des formations, on peut avancer l'hypothèse que les apprenants de FLS dans les Cours de langue pour immigrants au Canada (CLIC) ainsi que dans les cours de FLS financés par les gouvernements provinciaux ont un large éventail de profils. Ainsi, dans une même classe on peut retrouver des apprenants d'âges variés, de langues maternelles différentes, de divers niveaux de scolarisation ou encore de domaines d'expertise très différents. Ces classes peuvent aussi inclure des apprenants ayant des besoins en alphabétisation. Les formateurs nous ont donc rapporté qu'ils devaient composer avec une clientèle hétérogène qui avait des objectifs de vie et d'apprentissage bien différents.

Selon les principes établis lors du développement des échelles NCLC, énumérés dans la section précédente de cet article, les cours basés sur les NCLC doivent nécessairement commencer par une analyse des besoins afin de choisir des thèmes qui conviendront le mieux aux apprenants. Il faut également que les ressources développées puissent s'adapter à cette clientèle hétérogène.

Évaluation linguistique basée sur le portfolio. À la fin des années 2000, deux rapports portant sur les cours de FLS pour immigrants adultes financés par le gouvernement fédéral ont mis en lumière la nécessité d'une évaluation plus authentique et planifiée par les 
formateurs (Miaskovsky, 2008 ; Nagy et Stewart, 2009). Le projet de recherche qui a découlé de cette recommandation a abouti au protocole d'évaluation linguistique basée sur le portfolio (ELBP).

Inspirée par les recherches récentes sur le portfolio (p. ex. Johnson, Mims-Cox et Doyle-Nichols, 2006 ; Little, 2002 ; O'Rourke et Carson, 2010), l'ELBP est une démarche menée conjointement par le formateur et l'apprenant tout au long du cycle d'apprentissage ou d'enseignement. Les deux fixent ensemble des objectifs d'apprentissage, recueillent de nombreuses traces de la compétence linguistique dans divers contextes sur une période de temps déterminée, analysent ces traces et reviennent sur les progrès accomplis. Ces traces sont les tâches langagières réalisées par l'apprenant et qui illustrent ses progrès et ses défis. Cette démarche incite l'apprenant à devenir autonome, activement engagé dans son apprentissage ainsi que conscient et responsable de sa progression. Dans l'esprit d'une vision holistique de l'enseignement-apprentissage-évaluation, l'ELBP facilite l'acquisition de connaissances et de compétences métacognitives que l'apprenant peut mettre en pratique dans différents aspects de sa vie, hors de la salle de classe. C'est « un document écrit dans lequel les acquis de formation d'une personne sont définis, démontrés et articulés, en fonction d'un objectif » (Legendre, 2005, p. 1059).

Concrètement, dans des programmes de FLS en milieu minoritaire, le portfolio est un cartable que l'on offre à chaque nouvel apprenant. Il se présente en trois versions : apprenants en alphabétisation, apprenants débutants, et apprenants intermédiaires et avancés. Le niveau de langue de chaque version est modulé en fonction des capacités linguistiques de chaque groupe-cible.

L'utilisation d'un portfolio peut être variée et répondre à des objectifs divers. Comme mentionné auparavant, l'ELBP est à la fois un outil d'évaluation et d'apprentissage. Il peut servir aussi à « amener chaque élève à prendre conscience de ses apprentissages, à faire constamment le point sur ce qu'il maîtrise déjà et sur ce qu'il lui reste à apprendre, à envisager des stratégies pour combler des écarts, à se donner des objectifs ou des buts » (Scallon, 2004, p. 305). Enfin, le portfolio est utilisé pour faire un bilan de pratiques pédagogiques et guider l'enseignant dans le choix de stratégies qui permettent aux apprenants de progresser davantage.

En contexte minoritaire, l'ELBP est une ressource inestimable, car l'apprenant y trouve des repères francophones utiles pour parler français et pour vivre en français. Le portfolio fait le lien entre les besoins de l'apprenant, une progression langagière personnelle et la communauté francophone dont il souhaite se rapprocher. On y trouve entre autres une liste des sites Web des organismes communautaires francophones pour chaque province canadienne où l'on enseigne aux adultes avec les NCLC. On y trouve également un espace dans lequel l'apprenant peut intégrer des informations sur sa province au niveau géographique, gouvernemental, ou encore au niveau des moyens de transport disponibles, et cetera.

Le CLIC en ligne. Comme tout adulte, un apprenant immigrant a souvent des conditions de vie exigeantes et doit concilier travail ou recherche d'emploi, et famille. Faire des études dans ce contexte est un défi. Toujours dans la logique d'une recherche design en phase avec la diversité des besoins et des contraintes (Wang et Hannafin, 2005), il est indispensable aujourd'hui d'élargir les possibilités d'accès à l'apprentissage pour ces immigrants. Des ressources de formation en ligne s'avèrent indispensables, en particulier en dehors de grands centres urbains. 
Le projet CLIC en ligne (acronyme pour Cours de langue pour les immigrants au Canada en ligne) est un cours asynchrone au niveau des activités de compréhension de l'oral, de compréhension de l'écrit et d'expression écrite. L'expression orale est exploitée lors de classes virtuelles et dans le cadre d'un rendez-vous individuel hebdomadaire avec un formateur. Les activités tiennent compte des exigences des différents niveaux des NCLC. Des unités thématiques sont proposées à l'apprenant : la famille, les relations, les loisirs, le logement, le déplacement, les transports, la consommation, les services, la santé, la citoyenneté, l'éducation, l'emploi, le Canada. Ces thèmes sont pertinents pour tous les nouveaux arrivants au Canada, mais ont été adaptés aux besoins des apprenants de FLS en milieu minoritaire. Par exemple, il était important d'aider ces immigrants à repérer les services francophones de soutien à l'établissement offerts dans les régions où ils vivent.

Le projet CLIC en ligne a exigé de l'équipe de développement de répondre au mieux aux besoins particuliers des apprenants. Le modèle de formation asynchrone a été choisi pour encourager les apprenants à continuer leur apprentissage. L'alternance entre l'apprentissage autonome et les interactions avec un formateur s'est avérée être un facteur de motivation important.

\section{Outils et ressources pour formateurs en contexte d'intégration des immigrants adultes}

Les formateurs de FLS aux adultes en milieu minoritaire travaillent souvent seuls ou en très petites équipes, loin de grands centres urbains dotés d'un accès facile aux ressources pédagogiques. De plus, il n'existe pas de certification. Leur formation n'est donc pas standardisée. Il en résulte une grande variabilité de parcours qui mènent les formateurs à exercer leur métier. Dans ce contexte, les formations aux NCLC et leurs outils connexes constituent la partie intégrante de la démarche pédagogique : elles permettent de standardiser les pratiques et elles fournissent des occasions d'apprentissage pour les enseignants, contribuant ainsi à l'essor d'une communauté professionnelle. Dans la suite de l'article, nous présentons deux exemples de formations développées dans cette perspective : la formation à l'utilisation du portfolio et les Lignes directrices de l'Ontario.

La formation à l'évaluation linguistique basée sur le portfolio. L'utilisation du portfolio étant obligatoire dans les programmes de FLS en milieu minoritaire financés par le gouvernement fédéral et par certains gouvernements provinciaux, il était important d'offrir aux formateurs toutes les ressources nécessaires pour comprendre l'utilité et les enjeux de cet outil. Former ceux-ci était donc une étape indispensable pour les soutenir dans l'implantation de l'ELBP et pour assurer une harmonisation des pratiques à travers différents centres d'enseignement. Pour ce faire, un cours en ligne a été créé en 2016 pour des formateurs nommés champions. Ceux-ci ont été sélectionnés en fonction entre autres de leur niveau de formation pédagogique et de leur expérience d'enseignement. Une fois les champions formés, ils sont devenus, sur le terrain, des personnes ressources pour leurs collègues qui commençaient le cours un peu plus tard. Des classes virtuelles pour les champions et des rencontres en présentiel pour l'ensemble des enseignants font également partie de cette formation. Celle-ci mise sur le travail collaboratif et s'appuie sur des activités et projets à réaliser, sur des lectures et sur des discussions thématiques.

Toutefois, la formation ne se limite pas seulement à l'utilisation du portfolio. Elle permet de reprendre les grandes notions et les principes utiles à un enseignement avec les NCLC : l'analyse des besoins, l'enseignement basé sur la tâche, et cetera. Son objectif est 
ainsi de permettre aux formateurs d'exploiter l'ELBP de façon autonome et de répondre aux exigences en termes de reddition de compte.

\section{Les Lignes directrices de l'Ontario pour le programme de formation}

linguistique pour adultes ne donnant pas droit à des crédits. Les Lignes directrices, développées pour le compte du ministère de l'Immigration de l'Ontario, prennent la forme d'un portail interactif qui comprend un document sur les principes fondateurs de la formation linguistique pour adultes et un outil de planification en ligne destiné aux intervenants de FLS et à ceux d'anglais langue seconde (ALS).

L'outil de planification en ligne est appelé Quartz, en référence à l'améthyste, variété de quartz et minéral, symbole de l'Ontario. S'appuyant sur les NCLC et les CLB, les recherches et enquêtes sur le FLS/ALS au Canada et dans le monde ainsi que sur la situation du français et de l'anglais en Ontario, les équipes de développement ont préparé un document qui met de l'avant huit principes fondateurs :

1. Les programmes de FLS sont centrés sur l'apprenant.

2. Les programmes de FLS sont élaborés en fonction de besoins précis.

3. Les programmes de FLS appliquent les principes de l'approche communicative.

4. Les objectifs d'apprentissage sont explicites.

5. L'évaluation permet de vérifier l'atteinte des objectifs d'apprentissage et donne des informations sur le cours.

6. L'enseignement du FLS s'appuie sur une expertise reconnue et prouvée.

7. Les programmes de FLS facilitent l'apprentissage du français en contexte minoritaire et l'intégration des immigrants dans les communautés francophones.

8. Les programmes de FLS permettent une reddition de compte à la communauté.

Quartz a été conçu de manière à faciliter l'application de ces principes et à assurer l'harmonisation des pratiques. Il permet de planifier un cours, à partir des informations les plus générales (titre du cours, habiletés visées, horaire du cours, etc.) jusqu'aux informations les plus précises (contenus langagiers ciblés, déroulement d'une séquence d'enseignement, etc.). Il permet de planifier à la fois l'enseignement et l'évaluation.

Cet outil propose une structure de formation à trois niveaux : cours, unité, leçon. Un cours comprend un ensemble d'unités qui comprennent, à leur tour, des groupes de leçons. Il est possible de prévoir la planification seulement au niveau du cours, de l'unité ou de la leçon. En revanche, si l'on souhaite planifier la formation du début à la fin, les niveaux de planification du cours, des unités et des leçons sont interdépendants. Cela signifie qu'une information sélectionnée au niveau du cours, par exemple, est conservée dans la suite de la planification et permet ainsi un filtrage des données.

Quartz permet de choisir des informations parmi un ensemble de propositions ou de les rédiger soi-même, et ceci à plusieurs niveaux, par exemple :

- des besoins de l'apprenant dans la vie courante ;

- des thèmes ;

- des tâches que l'apprenant a besoin de réaliser dans la vie courante ;

- des tâches de communication réalisables en classe et qui vont aider l'apprenant à réaliser les tâches de la vie réelle ; 
- des tâches d'évaluation permettant à l'apprenant de montrer dans quelle mesure il est capable de réaliser une tâche reproduisant les exigences d'une tâche de la vie réelle.

Le résultat de la planification se présente sous la forme d'un document imprimable et qui peut être également archivé. Il regroupe toutes les informations sélectionnées ou rédigées.

L'outil de planification offre également une banque de ressources permettant aux intervenants de s'informer sur différents sujets (l'analyse des besoins, l'enseignement par la tâche, l'évaluation par la tâche, etc.) ou d'utiliser des documents mis à disposition pour leur enseignement : exemple de formulaires d'analyse de besoins, de formulaires d'évaluation ou encore d'unités complètes.

Les formateurs de FLS qui utilisent les NCLC, contrairement à ceux du réseau anglophone, ne bénéficient pas aujourd'hui d'une formation accréditée. Une telle formation joue pourtant un rôle crucial dans la professionnalisation des intervenants et dans l'harmonisation des pratiques d'enseignement et d'évaluation. Sans pouvoir pallier ce manque, Quartz offre toutefois un cadre pour l'enseignement et de nombreuses ressources et exemples d'application qui tiennent compte de la situation du français en milieu minoritaire. Par exemple, une banque de ressources de tâches authentiques adaptées à ce contexte a été créée. Ces tâches sont regroupées sous le thème « Minorités francophones » et classées selon des sous-thèmes et des besoins pertinents pour un apprenant de FLS souhaitant s'intégrer dans la communauté francophone où il vit. Il s'agit, par exemple, de la vie des minorités francophones (comprendre leur histoire, participer à la vie culturelle), des droits linguistiques (les comprendre et défendre le droit de vivre en français) ou des services en français (les connaître et utiliser).

\section{Une proposition pédagogique raisonnée en FLS pour immigrants adultes en milieu minoritaire : acquis, défis et perspectives}

Le présent article fait état d'une proposition pédagogique en FLS pour immigrants adultes en milieu minoritaire. Tout d'abord, la revue de la problématique a permis d'entrevoir l'importance de la formation langagière en FLS pour immigrants adultes qui vivent dans les provinces canadiennes anglophones. En effet, pour assurer la vitalité des communautés francophones minoritaires, il est essentiel qu'une certaine proportion de cette population s'intègre en français. En même temps, la revue de la problématique a permis de constater que les communautés francophones minoritaires sont dispersées à travers le Canada et il n'est donc pas facile d'assurer un système de formation pancanadien. Et pourtant, les différents acteurs de la formation en FLS pour immigrants adultes hors Québec manifestent le besoin d'un tel système.

Pour répondre donc à la fois aux besoins des communautés francophones minoritaires et à ceux des immigrants qui souhaitent s'y installer, le gouvernement fédéral a commandité le développement du référentiel Niveaux de compétence linguistique canadiens dont l'objectif principal était d'aider la standardisation des pratiques de l'enseignement du FLS en contextes minoritaires.

La version 2012 de ce référentiel a été le résultat d'un projet de recherche de type recherche design et comprend (a) le cadre théorique pour l'enseignement et l'évaluation du FLS en milieu minoritaire, basé sur les travaux de Bachman (1990 ; Bachman et Palmer, 
1996, 2010) cités antérieurement ; (b) les échelles de compétence et (c) les principes d'une pédagogie du FLS pour adultes immigrants en milieu minoritaire (une approche centrée sur les apprenants, les compétences, les tâches et les contextes).

Depuis 2012, une panoplie d'outils et de ressources pour les enseignants et les apprenants de FLS en milieu minoritaire ont été développés. Seuls quelques-uns de ces outils ont été présentés ici, mais plusieurs autres peuvent être consultés sur le site dédié (www.language.ca) : des formations ciblées, des batteries de tests de classement et de rendement, des outils destinés à des clientèles spécifiques, par exemple, celles en alphabétisation ou celles en formation en milieu de travail, et cetera. Tous ces nouveaux outils s'appuient maintenant sur la conception commune de l'enseignement du FLS en milieu minoritaire qui a découlé de recherches menées pour construire le cadre théorique et les échelles de compétence des NCLC 2012. C'est dans ce contexte que nous souhaitons parler d'une « proposition pédagogique raisonnée ».

L'élaboration de cette proposition a fourni des acquis non négligeables à l'enseignement du FLS aux adultes immigrants en milieux minoritaires. Tout d'abord, l'harmonisation des pratiques à travers les centres d'enseignement dispersés sur le territoire du Canada a promu une communauté de pratique là où les enseignants avaient rarement l'occasion de côtoyer leurs collègues et où la clientèle immigrante était caractérisée par une grande mobilité à la fois géographique (d'une localité à une autre, d'une province à une autre, etc.) et sociale (d'un emploi à un autre, d'une profession à une autre, d'une communauté à une autre, etc.).

Ce système de formation a également été développé avec un grand souci de souplesse, autant pour les apprenants que pour les formateurs et pour les gestionnaires de programmes. Dans un premier temps, le besoin de souplesse des outils et des ressources a été exprimé par les acteurs sur le terrain : les immigrants adultes confrontés aux défis d'apprentissage d'une langue en contexte d'intégration sociale et professionnelle, les formateurs dont les parcours professionnels et les conditions de travail varient grandement, les gestionnaires aux prises avec une grande variabilité de la demande. Dans un deuxième temps, la recherche a fourni des solutions (ou des éléments de solution) à ce besoin de souplesse. Par exemple, les théories et les résultats de recherches en apprentissage des langues assisté par ordinateur ont permis de mettre en place des outils de formation en ligne, autant pour les apprenants (CLIC en ligne) que pour les enseignants (Lignes directrices).

Les besoins des communautés minoritaires, identifiés par des recherches menées pour le compte de ces communautés, ont également été pris en compte lors de développement des outils pour le FLS. Par exemple, selon un rapport récent du Commissariat aux langues officielles (2012), les ressources bilingues sont considérables, mais souvent invisibles, et les ressources et services francophones ont bien du mal à émerger dans un contexte où l'anglais domine largement. Dans ce contexte, il a été essentiel de les regrouper, de les classer et de les signaler aux formateurs et aux apprenants. Les banques de ces données ont été créées dans le cadre de projets tels que l'ELBP, mais ont besoin d'une mise à jour continuelle étant donné que ces ressources et services perdent rapidement de l'actualité. De la même façon, il a été primordial de créer des contenus adaptés à la situation des francophones au Canada qui ne peuvent pas utiliser le français de façon exclusive. Proposer des tâches authentiques est un défi continuel lors de développement d'outils pédagogiques pour ces contextes. 
En dépit de nombreux acquis, le travail d'harmonisation des pratiques d'enseignement du FLS aux adultes en contexte minoritaire est un projet qui demande du temps. Penser aux communautés francophones d'accueil est un bon point de départ pour comprendre ce qui manque et pour mieux cerner ce qu'il faut développer à l'avenir. De façon générale, le besoin d'un programme-cadre pour la formation linguistique des immigrants adultes basé sur les NCLC se fait de plus en plus criant. Il serait souhaitable que ce programme résulte d'une démarche de recherche, à l'instar des ressources et outils déjà existants.

Parmi d'autres défis majeurs, on peut nommer, en guise de conclusion, la recherche sur la pédagogie par compétences, sur la pédagogie par tâches et, en particulier, la conceptualisation de ce qu' est une tâche langagière authentique en milieu minoritaire, ou encore, la recherche sur certains publics cibles particuliers, tels le public en alphabétisation en FLS ou le public en formation FLS pour l'emploi en contextes bilingues. Le renforcement du rôle de la recherche dans ces contextes, au-delà même de l'amélioration des pratiques et des outils, est une condition incontournable afin d'assurer l'intégration des nouveaux arrivants à la francophonie canadienne.

La correspondance devrait être adressée à Morgan Le Thiec.

Courriel : le_thiec.morgan@uqam.ca

\section{Notes}

${ }^{1}$ On définit le terme immigrant adulte dans son acception adoptée par Statistique Canada : un résident ou citoyen canadien de plus de 16 ans né à l'étranger de parents non canadiens.

${ }^{2}$ Première langue officielle parlée de la personne (PLOP) est un concept précisé en 2009 dans le cadre de la Loi sur les langues officielles. Il désigne la langue officielle (le français ou l'anglais) que les citoyens et résidents canadiens privilégient pour fonctionner dans la société (interaction avec des structures gouvernementales, éducation, vie sociale, etc.). Pour les immigrants, il s'agit de la langue d'usage prioritaire hors du milieu familial ou de la communauté culturelle. Ce concept est utilisé comme variable dans les enquêtes de Statistique Canada.

${ }^{3}$ Dans la littérature anglo-saxonne, on utilise les termes educational design research (Van Den Akker, Gravemeijer, McKenney et Nieveen, 2006 ; Barab et Squire, 2004), design experiments (Brown, 1992 ; Cobb, Confrey, diSessa, Lehrer et Schaube, 2003) ou developmental research (Richey, Klein et Nelson, 2004). Dans cet article, nous avons opté pour la traduction de ces termes proposée par Basque (2015) : recherche design.

\section{Références}

Bachman, L. F. (1990). Fundamental considerations in language testing. Oxford, Royaume-Uni : Oxford University Press.

Bachman, L. F. et Palmer, A. (1996). Language testing in practice: Designing and developing useful language tests. New York, NY : Oxford University Press. 
Bachman, L. F. et Palmer, A. (2010). Language assessment practice: Developing language assessments and justifying their use in the real world . Oxford, Royaume-Uni : Oxford University Press.

Barab, S. et Squire, K. (2004). Design-based research: Putting a stake in the ground. Journal of the Learning Sciences, 13(1), 1-14.

Basque, J. (2015, octobre). Un modèle méthodologique de recherche-design (design-based research) pour favoriser l'innovation pédagogique en enseignement supérieur. Communication présentée au Colloque Cirt, Université de Sherbrooke, Canada. Repéré à http://rlibre.teluq.ca/737/1/Cirta2015_Basque_Un\%20mod $\% \mathrm{C} 3 \%$ A $81 \mathrm{e} \% 20 \mathrm{~m} \% \mathrm{C} 3 \%$ A 9 tho dologique\%20de\%20recherche-design_VF.pdf

Bournot-Trites, M. (2017). Design-based research methodology for establishing the Common Theoretical Framework and the CLB/NCLC Scales. Dans M. Jezak (dir.), Language is the key. The Canadian Language Benchmarks model. Ottawa, ON : Presses de l'Université d'Ottawa.

Bournot-Trites, M. et Barbour, R. (2012). Report on phases I and II of the validation of the Canadian Language Benchmarks and Niveaux de compétence linguistique canadiens. Ottawa, ON : Centre des niveaux de compétence linguistique canadiens.

Bournot-Trites, M., Barbour, R., Jezak, M., Stewart, G. et Blouin Carbonneau, D. (2015). Theoretical framework for the Canadian Language Benchmarks and Niveaux de compétence linguistique canadiens. Ottawa, ON : Centre des niveaux de compétence linguistique canadiens.

Brown, A. (1992). Design experiments: Theoretical and methodological challenges in creating complex interventions in classroom settings. The Journal of the Learning Sciences, 2(2), 141-178.

Cardinal, L., Gaspard, H. et Léger, R. (2015). The politics of language roadmaps in Canada: Understanding the Conservative government's approach to official languages. Canadian Journal of Political Science/Revue canadienne de science politique, 1-23. doi : 10.1017/S0008423915000517

Chong, M. D. (2015). L'immigration : un outil pour assurer la vitalité et l'épanouissement des communautés francophones en situation minoritaire : rapport du Comité permanent des langues officielles. Ottawa, ON, Chambre des communes Canada, $41^{\mathrm{e}}$ législature, $2^{\mathrm{e}}$ session.

Citoyenneté et Immigration Canada. (1996). Canadian Language Benchmarks: English as a second language for adults (working document, 1996). Ottawa, ON : Ministère des travaux publics et des services Canada.

Citoyenneté et Immigration Canada et Centre des niveaux de compétence linguistique canadiens. (2002). Standards linguistiques canadiens 2002 : français langue seconde pour adultes. Ottawa, $\mathrm{ON}$ : Ministère des travaux publics et des services Canada.

Citoyenneté et Immigration Canada et Centre des niveaux de compétence linguistique canadiens. (2006). Niveaux de compétence linguistique canadiens 2006 : français langue seconde pour adultes. Ottawa, $\mathrm{ON}$ : Citoyenneté et Immigration Canada.

Citoyenneté et Immigration Canada et Centre des niveaux de compétence linguistique canadiens. (2012). Niveaux de compétence linguistique canadiens : français langue seconde pour adultes. Ottawa, $\mathrm{ON}$ : Citoyenneté et Immigration Canada. 
Cobb, P., Confrey, J., diSessa, A., Lehrer, R. et Schauble, L. (2003). Design experiments in educational research. Educational Researcher, 32(1), 9-13.

Collins, A. (1992). Toward a design science of education. Dans E. Scanlon et T. O'Shea (dir.), New directions in educational technology. Berlin, Allemagne : Springer Verlag.

Commissariat aux langues officielles. (2012). Rapport annuel du Commissariat aux langues officielles 2011-2012. Ottawa, ON.

Corbeil, J.-P. (2014). Document de travail sur la définition statistique de la population de langue française au Canada. Ottawa, ON : Fédération des communautés francophones et acadienne (FCFA) du Canada.

Corbeil, J.-P., Grenier, C. et Lafreniere, S. (2006). Les minorités prennent la parole : résultats de l'Enquête sur la vitalité des minorités de langue officielle (produit $\mathrm{n}^{\mathrm{o}}$ 91-548-X au catalogue). Ottawa, ON : Statistique Canada.

Dancose, S. et Ricard, P. (2007). Perceptions des intervenants en français langue seconde sur l'utilisation des Niveaux de compétence linguistiques canadiens 2006, français langue seconde pour adultes dans leur contexte et milieu de travail. Ottawa, ON : Centre des niveaux de compétence linguistique canadiens.

Dede, C. (2004). If design-based research is the answer, what is the question? Journal of the Learning Sciences, 13(1), 105-114.

Design-Based Research Collective. (2003). Design-based research: An emerging paradigm for educational inquiry. Educational Researcher, 32(1), 5-8.

Elson, N. (2012). Final report on the benchmarking of the revised Niveaux de compétences linguistique canadiens (NCLC) by the expert panel and field practitioners. Ottawa, $\mathrm{ON}$ : Centre for the Canadian Language Benchmarks.

Fraser, G. et Boileau, F. (2014). Agir maintenant pour l'avenir des communautés francophones : pallier le déséquilibre en immigration. Ottawa, ON : Ministère des travaux publics et des services Canada.

Gouvernement du Canada. (2003). Cadre stratégique pour favoriser l'immigration au sein des communautés francophones en situation minoritaire. Ottawa, ON : Gouvernement du Canada.

Gouvernement du Canada. (2006). Plan stratégique pour favoriser l'immigration au sein des communautés francophones en situation minoritaire. Ottawa, ON : Gouvernement du Canada.

Gouvernement du Canada. (2008). Feuille de route pour la dualité linguistique 2008-2013. Ottawa, ON : Gouvernement du Canada.

Gouvernement du Canada. (2009). Plan d'action 2009-2013 : mise en ouvre de l'article 41 de la Loi sur les langues officielles. Ottawa, ON : Gouvernement du Canada.

Gouvernement du Canada. (2013). Feuille de route pour les langues officielles du Canada 2013-2018 : éducation, immigration, communautés. Ottawa, ON : Gouvernement du Canada.

Harvey, L. (2008). Les initiatives canadiennes d'assurance de la qualité vues dans le contexte international. Ottawa, ON : Conseil des ministres de l'éducation (Canada). Repéré à http://cmec.ca/Publications/Lists/Publications/Attachments/117/2008-05lee-harvey.fr.pdf

Harvey, S. et Loiselle, J. (2009). Proposition d'un modèle de recherche développement. Recherches qualitatives, 28(2), 95-117. 
Houle, R., Pereira, D. et Corbeil, J.-P. (2015). Portrait statistique de la population immigrante de langue française à l'extérieur du Québec (1991 à 2011). Ottawa, $\mathrm{ON}$ : Citoyenneté et Immigration Canada.

Jezak, M. et Carrasco, E. (2017). Integration trajectories of adult (im)migrants in minority and minoritized contexts: Ottawa and Barcelona. Dans J.-C. Beacco, D. Little, H.-J. Krumm et $\mathrm{P}$. Thalgott (dir.), The linguistic integration of adult migrants: Some lessons from research. Berlin, Allemagne : De Gruyter Mouton/Council of Europe.

Johnson, R., Mims-Cox, J. S. et Doyle-Nichols, A. (2006). Developing portfolios in education: A guide to reflection, inquiry, and assessment. Londres, Royaume-Uni : Sage.

Kelly, A. (2003). Research as design. Educational Researcher, 32(1), 3-4.

Klieme, E., Avenarius, H., Blum, W., Döbrich, P. et Vollmer, H. J. (2004). Le développement de standards nationaux de formation. Bonn, Allemagne : Ministère fédéral de l'Éducation et de la Recherche.

Legendre, R. (2005). Dictionnaire actuel de l'éducation. Montréal, PQ : Guérin.

Little, D. G. (2002). The European language portfolio: Structure, origin, implementation and challenges. Language Teaching, 35, 182-189.

Loiselle, J. et Harvey, S. (2007). La recherche développement en éducation : fondements, apports et limites. Recherches qualitatives, 27(1), 40-59.

Martel, L. (2015). Changements récents dans les tendances démographiques au Canada. Regards sur la société canadienne (produit $\mathrm{n}^{0}$ 75-006-X au catalogue). Ottawa, ON : Statistique Canada.

Miaskovsky, L. (2008). The feasibility and way forward for a standardized exit assessment and test for newcomers in LINC training (Internal report). Ottawa, ON :

Citoyenneté et Immigration Canada.

Nagy, P. et Stewart, G. (2009). Research study on potential approaches to second language assessment. Ottawa, ON : Citoyenneté et Immigration Canada.

O’Rourke, B. et Carson, L. (dir.). (2010), Language learner autonomy: Policy, curriculum, classroom. Oxford, Royaume-Uni : Peter Lang.

Organisation de Coopération et de Développement Économiques. (2015). Perspectives des politiques de l'Éducation 2015, des réformes en marche. Paris, France : Organisation de Coopération et de Développement Économiques.

Quell, C. (2008). Recherches sur la nouvelle diversité des communautés francophones en situation minoritaire. Dans C. Belkhodja (dir.), Thèmes canadiens : immigration et diversité au sein des communautés francophones en situation minoritaire. Montréal, PQ : Association d'études canadiennes.

Reeves, T., Herrington, J. et Oliver, R. (2005). Design research: A socially responsible approach to instructional technology research in higher education. Journal of Computing in Higher Education, 16(2), 97-116.

Richey, R., Klein, J. et Nelson, W. (2004). Developmental research: Studies of instructional design and development. Dans D. Jonassen (dir.), Handbook of research for educational communications and technology ( $2 \mathrm{e}$ éd.). Bloomington, IN : Association for Educational Communications \& Technology.

Scallon, G. (2004). L'évaluation des apprentissages dans une approche par compétences. Montréal, PQ : ERPI.

Van Den Acker, J., Gravemeijer, K., McKeineey, S. et Nieveen, N. (2006). Educational design research. New York, NY : Routledge. 
Van der Maren, J. M. (1995). Méthodes de recherche pour l'éducation. Montréal, PQ : Les Presses de l'Université de Montréal.

Wang, F. et Hannafin, M. (2005). Design-based research and technology-enhanced learning environments. Educational Technology Research and Development, 53(4), 5-23. doi : 10.1007/BF02504682 